\title{
A nonnegative Matrix Tri-Factorization Technique for Recommendation in Microblog
}

\author{
Guoying Zhang ${ }^{1, a}$, Guanghui Cai ${ }^{2, b}, \mathrm{Hao} \mathrm{Wu}^{1, \mathrm{c}}$ and Shuwen Zheng ${ }^{1, \mathrm{~d}}$ \\ ${ }^{1}$ School of Information Science and Engineering, Yunnan University, Kunming 650091, China; \\ ${ }^{2}$ School of Resource Environment and Earth Science, Yunnan University, Kunming 650091, China. \\ ajqueebi@126.com, bghcai@ynu.edu.cn, chaowu@ynu.edu.cn, ${ }^{d} 463580488 @ q q . c o m$
}

Keywords: Micro-blogging communities, Recommendation system, Demographic information, Genre information.

\begin{abstract}
Nowadays, more and more users share news and information in micro-blogging communities such as Twitter, Tumblr or Plurk. With the increasing number of registered users in this kind of sites, finding relevant and reliable sources of information becomes essential. For recommending sufficient information for a user, it is important to extract automatically user's interest with accuracy and to collect new information which interests and attracts the user. To address this problem, we propose a social matrix tri-factorization model with two auxiliary matrices to improve the accuracy of recommendation in microblog. We construct two matrices on the users and items respectively, to exploit the user's demographic information and item's genre information which play an important role in microblog recommendation system. Experimental results show that our method achieves a higher performance and provide more effective results than the state-of -art methods especially when recommending informations. We also show that out model captures user interests more precisely and gives better recommendation interpretability.
\end{abstract}

\section{Introduction}

Microblog, such as Twitter and Google+, has become a popular Internet service. Essentially, microblog enables an easy and lightweight way of communication, which allows people to write short messages and then broadcast and share them through the participating online social networks. A large number of microblog users, together with the low cost and effort required for sharing messages, produce massive and fast growing information in microblog, giving rise to the problem of information overload. It thus becomes a great challenge for microblog users to find interesting information. Surely, an effective recommending information sources service can assist information seekers to discover and connect with other users in a satisfactory manner. Promisingly, it helps increase the linkages in the social networks while significantly improving user experience in microblog. As a result, users do not leave microblog or become inactive, which is crucial to the survival and growth of microblog site in the long run [1].

Recently, the model based approach for recommendation in social rating networks has been investigated [2][3].Matrix factorization is a popular method in traditional item recommendation, which finds latent features for users and items by factorizing the observed user-item rating matrix and makes latent features for further predictions. However, these methods do not consider the external information such as user's demographic information, item's genre information or propagation of trust which plays very important role in microblog recommendation systems. For instance, users with similar tags may have similar interest in microblogs. Microblogs of the same genre may be liked similarly by users. And a user usually asks his/her trusted person for the recommendation on microblogs. In this paper, we also use a matrix factorization based model for recommendation in social networks, called Social Nonnegative Matrix Tri-Factorization (SocialNMTF). We construct two matrices on the users and items respectively, so our methods has the ability to make use of the user's demographic information and item's genre information which is used by microblog recommendation system. Due to the use of these information, our method is able to find more interpretable low-dimensional representations for users and microblogs, which 
consequently improve the recommendation performance. Experimental results show that the proposed methods perform superior to the art collaborative filtering methods.

\section{The Social Nonnegative Matrix Tri-Factorization Model}

Nonnegative Matrix Tri-Factorization have been proposed in [4].Using the similar method, suppose we have $M$ microblogs and $N$ users, $X \in R_{+}^{N \times M}$ is the target matrix, where $X_{i j}$ represents the sums of repostscount and commentscount of user $i$ to the microblog $j . \mathrm{Y} \in \mathrm{R}^{\mathrm{N} \times \mathrm{M}}$ is an indicator matrix where $Y_{i j}=1$ if user $i$ reposted or commented microblog $j$ and $Y_{i j}=0$ otherwise. In matrix factorization based model, we usually seek two low-rank matrices $\mathrm{U} \in \mathrm{R}_{+}^{\mathrm{N} \times d}$ and $\mathrm{V} \in \mathrm{R}_{+}^{\mathrm{M} \times d}$. The row vector $u_{i}, 1 \leq i \leq N$ and $v_{j,}, 1 \leq j \leq M$ represent the low-dimensional representations of users and microblogs respectively.

We denote by $\mathrm{f}_{i}^{U}$, the feature vector which characterizes the demographic information of user $i$,then the assumption can be embodied by the adjacency matrix on user as follows,

$$
W_{i j}^{U}=\operatorname{sim}\left(\mathrm{f}_{i}^{U}, \mathrm{f}_{j}^{U}\right)
$$

Where $\operatorname{sim}\left(\mathrm{f}_{i}^{U}, \mathrm{f}_{j}^{U}\right)$ is the similarity between the feature vectors of user $i$ and user $j$.In our experiment, we use cosine distance for simplicity. Then we denotes $D_{i i}^{U}=\sum_{j} W_{i j}^{U}$ is a diagonal matrix, and $\mathrm{L}_{U}=D^{U}-W^{U}$.

Similarly, we denote by $\mathrm{f}_{i}^{V}$, the feature vector which characterizes the genre information of microblog $i$,then the assumption can be embodied by the adjacency matrix on microblog as follows,

$$
W_{i j}^{V}=\operatorname{sim}\left(\mathrm{f}_{i}^{V}, \mathrm{f}_{j}^{V}\right)
$$

Our method aims to find three nonnegative matrices $\mathrm{U} \in \mathrm{R}_{+}^{\mathrm{N} \times m}, \mathrm{~S} \in \mathrm{R}_{+}^{m \times d}$ and $\mathrm{V} \in \mathrm{R}_{+}^{M \times d}$ which minimize the following objective

$$
J=\left\|\mathrm{Y} \odot\left(\mathrm{X}-\mathrm{USV}^{T}\right)\right\|_{F}^{2}+\lambda \operatorname{tr}\left(\mathrm{U}^{T} \mathrm{~L}_{U} \mathrm{U}\right)+\mu \operatorname{tr}\left(\mathrm{V}^{T} \mathrm{~L}_{V} \mathrm{~V}\right) \quad \text { s.t. } \quad \mathrm{U} \geq 0, \mathrm{~S} \geq 0, \mathrm{~V} \geq 0
$$

Where $\odot$ is Hadamard product(element-wise product). \|\|$_{F}$ is Frobenius norm, $\mathrm{Y}$ is the indicator matrix, $\operatorname{tr}(\cdot)$ denotes the matrix trace, $\lambda, \mu \geq 0$ are regulation parameters balancing the reconstruction error of matrix factorization. Optimizing Eq. (3) with respect to $S$ is equivalent to optimizing

$$
L(S)=\left\|\mathrm{Y} \odot\left(\mathrm{X}-\mathrm{USV}^{T}\right)\right\|_{F}^{2} \text { s.t. } \mathrm{S} \geq 0
$$

The derivative of $\mathrm{L}(\mathrm{S})$ with respect to $\mathrm{S}$ is

$$
\frac{\partial L(\mathrm{~S})}{\partial \mathrm{S}}=-2 \mathrm{U}^{T}(\mathrm{Y} \odot \mathrm{X}) \mathrm{V}+2 \mathrm{U}^{T}\left(\mathrm{Y} \odot\left(\mathrm{USV}^{T}\right)\right) \mathrm{V}
$$

Using the Karush-Kuhn-Tucker complementary condition[5] for the nonnegativity of S, we set $\left[-2 \mathrm{U}^{T}(\mathrm{Y} \odot \mathrm{X}) \mathrm{V}+2 \mathrm{U}^{T}\left(\mathrm{Y} \odot\left(\mathrm{USV}^{T}\right)\right) \mathrm{V}\right]_{i j} \mathrm{~S}_{i j}=0$

Eq. (6) leads to the following updating formula

$$
\mathrm{S}_{i j} \leftarrow \mathrm{S}_{i j} \sqrt{\frac{\left[\mathrm{U}^{T}(\mathrm{Y} \odot \mathrm{X}) \mathrm{V}\right]_{i j}}{\left[\mathrm{U}^{T}\left(\mathrm{Y} \odot\left(\mathrm{USV}^{T}\right)\right) \mathrm{V}\right]_{i j}}}
$$

Optimizing Eq. (3) with respect to $U$ is equivalent to optimizing

$$
L(\mathrm{U})=\left\|\mathrm{Y} \odot\left(\mathrm{X}-\mathrm{USV}^{T}\right)\right\|_{F}^{2}+\lambda \operatorname{tr}\left(\mathrm{U}^{T} \mathrm{~L}_{U} \mathrm{U}\right) \text { s.t. } \mathrm{U} \geq 0
$$

The derivative of $\mathrm{L}(\mathrm{U})$ with respect to $\mathrm{U}$ is

$$
\frac{\partial L(\mathrm{U})}{\partial \mathrm{U}}=-2 \mathrm{Y} \odot \mathrm{XVS}^{T}+2 \mathrm{Y} \odot\left(\mathrm{USV}^{T}\right) \mathrm{VS}^{T}+2 \lambda \mathrm{L}_{U} \mathrm{U}
$$

Using the Karush-Kuhn-Tucker complementary condition for the nonnegativity of U, we get

$$
\left.\left[-\mathrm{Y} \odot \mathrm{XVS}^{T}+\mathrm{Y} \odot\left(\mathrm{USV}^{T}\right)\right) \mathrm{VS}^{T}+\lambda \mathrm{L}_{U} \mathrm{U}\right]_{i j} \mathrm{U}_{i j}=0
$$

Since $\mathrm{L}_{U}$ may take any signs, we decompose it as $\mathrm{L}_{U}=\mathrm{L}_{U}^{+}-\mathrm{L}_{U}^{-}$, then

$$
\left.\left[-\mathrm{Y} \odot \mathrm{XVS}^{T}+\mathrm{Y} \odot\left(\mathrm{USV}^{T}\right)\right) \mathrm{VS}^{T}+\lambda \mathrm{L}_{U}^{+} \mathrm{U}-\lambda \mathrm{L}_{U}^{-} \mathrm{U}\right]_{i j} \mathrm{U}_{i j}=0
$$


Eq. (11) leads to the following updating formula

$$
\begin{aligned}
& U_{i j} \leftarrow U_{i j} \sqrt{\frac{\left[\mathrm{Y} \odot \mathrm{XVS}^{T}+\lambda \mathrm{L}_{U}^{-} \mathrm{U}\right]_{i j}}{\left[\mathrm{Y} \odot\left(\mathrm{USV}^{T}\right) \mathrm{VS}^{T}+\lambda \mathrm{L}_{U}^{+} \mathrm{U}\right]_{i j}}} \\
& \mathrm{~V}_{i j} \leftarrow \mathrm{V}_{i j} \sqrt{\frac{\left[(\mathrm{Y} \odot \mathrm{X})^{T} \mathrm{US}+\mu \mathrm{L}_{V}^{-} \mathrm{V}\right]_{i j}}{\left[\mathrm{Y} \odot\left(\mathrm{USV}^{T}\right)^{T} \mathrm{US}+\mu \mathrm{L}_{V}^{+} \mathrm{V}\right]_{i j}}}
\end{aligned}
$$

\section{Experiments}

The experiment data we used in this study was downloaded from datatang.com[6], which, similar to Twitter, allows users to follow with each other. It consists of demographic information(e.g. gender and tags) of users and genre of microblogs. In detail, we use 2 dimensional feature vector to characterize the user's gender, that is, if the user is male, then the first feature is 1 while the second is 0, and vice versa. There are totally 12 tags: Meizu, Xiaomi, Rockets, Jeremy Lin, Evergrande, Korean drama, smog, house prices, deskmate of you, civil servants, corrupt official, genetically modified, other and none. So we use a 12 dimensional feature vector to describe the user's tags. We concatenate the 2 feature vectors mentioned above together to get a totally 14 dimensional feature vector $\mathrm{f}_{i}^{U}$ for user $i$.On the other hand, we extract 30 topics from all microblogs, there are totally 42 genres of microblogs. Likewise, we use a 42 dimensional feature vector $f_{i}^{V}$ for microblog $i$. We also evaluate the impact of neighborhood information of users and items on this data set.

Accuracy is one property of recommender system which characterizes whether generated recommendations accurately match users interests/preferences or not. Along with the development of recommendation techniques, various metrics have been employed for measuring the accuracy of recommendations including statistical accuracy metrics and decision-support measures [7]. Since we focus on recommending top- $\mathrm{N}$ items instead of rating-prediction of items, Precision@N and Recall@N are selected to evaluate the recommendation accuracy. Given a rank list of recommended items, Precision@N is the fraction of recommended items that are relevant in the top-N position (see Eq. (13)),

$$
\text { Pre@N= } \frac{\mid \text { relevant items }\} \cap\{\text { topN } \text { items }\} \mid}{N}
$$

While Recall@N is the fraction of relevant items returned in the top- $\mathrm{N}$ position, to the true number of relevant items that should have been returned (see Eq. (14)),

$$
\operatorname{Rec} @ N=\frac{\mid\{\text { relevant items }\} \cap\{\text { top } N \text { items }\} \mid}{\mid \text { relevant items }\} \mid}
$$
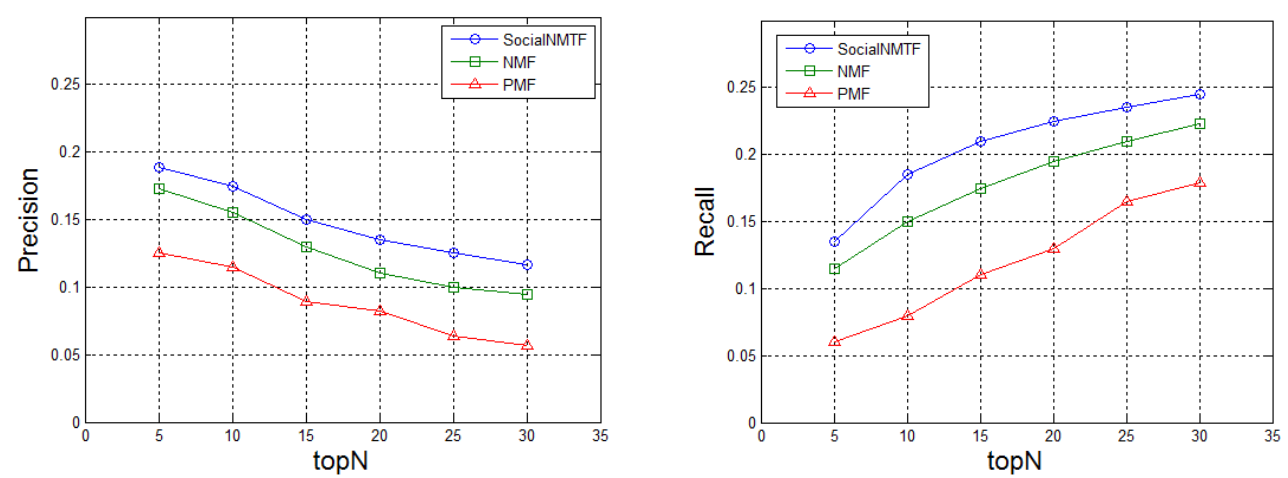

Fig. 1 Average precision and recall of different methods. 
Finally, we leveraged other baseline methods, such as NMF and PMF, to complete the ranking task on our proposed features. Due to space restriction, Figure 1 merely shows average precision and recall for each baseline method along with proposed method in this paper. From the above, compared to other baseline methods, our methods achieve better performance on both Precision and Recall, telling that SocialNMF can achieve higher ranking accuracy. Also, SocialNMF preserve the explainability of memory-based CF, and enable to give users a reason for their ranking.

\section{Conclusion}

In the microblogging systems, thousands of tweets are submitted per second and it is difficult for users to locate their interested ones. So exploiting the information hidden in the microblog to recommend informations to users precisely has become very important. To address this problem, in this paper we propose a SocialNMTF microblog recommendation. We construct two auxiliary matrices on the users and items respectively, to exploit the user's demographic information and item's genre information. Our key idea is to overcome the sparseness of the target data with complementary data. Experiments on microblog data sets demonstrate that the proposed methods outperform many state of the art collaborative filtering methods. We also confirmed that the estimated bases of the proposed methods are reasonably intuitive and easy to understand.

\section{References}

[1] P. Gupta, A. Goel, J. Lin, et al., "WTF: the who to follow service at twitter," in Proceedings of the 22nd International World Wide Web Conference Committee (WWW '13), 2013, 505-514.

[2] H. Ma, I. King, and M. R. Lyu. Learning to recommend with social trust ensemble. In SIGIR 2009, 2009, 203-210.

[3] H. Ma, H. Yang, M. R. Lyu, and I. King. Sorec: social recommendation using probabilistic matrix factorization. In CIKM 2008 ACM, 2008, 931-940.

[4] Gu Q, Zhou J, Ding C H, et al. Collab orative Filtering: Weighted Nonnegative Matrix Factorization Incorporating User and Item Graphs[J]. SIAM International Conference on Data Mining, 2010, 199-210.

[5] Stephen Boyd and Lieven Vandenberghe, Convex optimization, Cambridge University Press, Cambridge, 2004.

[6] http://www.datatang.com/data/46758/

[7] J. Herlocker, J. Konstan, L. Terveen, J. Riedl, Evaluating collaborative filtering recommender systems, ACM Trans. Inf. Syst. 22 (2004) 5-53. 